\title{
Generalized Traffic Flow Model for Multi-Services Oriented UAV System
}

\author{
Abderrahmane Abada ${ }^{1}$, Bin Yang ${ }^{1}$, and Tarik Taleb ${ }^{1,2}$ \\ ${ }^{1}$ School of Electrical Engineering, Aalto University, Finland \\ ${ }^{2}$ Centre for Wireless Communications, University of Oulu, Finland
}

\begin{abstract}
Unmanned Aerial Vehicles (UAVs) are opening up new opportunities for extensive applications. The traffic flow model is critical to evaluate the traffic needs of various applications in designing and deploying UAV system. However, the traffic flow model has not been explored in multi-services oriented UAV system. To this end, this paper proposes a general traffic flow model for multi-services orientated UAV system. Under such a model, the network services are first categorized into three subsets, each corresponding to one of telemetry, Internet of Things (IoT), and streaming data. According to the Pareto distribution, all UAVs are further partitioned into three subgroups relying on their network usages. We can measure the packet arrival rate for the nine segments, each of which represents one map relationship between a services subset and a UAV subgroup. Therefore, we can also obtain the number of packets for each network service and total data size. Simulation results are presented to illustrate that the number of packets and the data size predicted by our traffic model can well match with these in real scenarios with different network services.
\end{abstract}

Index Terms-UAV system, multi-services, traffic flow.

\section{INTRODUCTION}

W IRELESS communications with assistance of unmanned aerial vehicles (UAVs) have been identified as a key technology in the next generation wireless systems due to their promising advantages of low cost and fast deployment, flexible mobility, large area coverage and line-of-sight links [1]. Recently, UAVs are widely used in search and rescue operations, firefighting, agriculture, mapping, surveying and Internet of Things (IoT) [2], [3], [4], [5]. Equipped with wireless devices, UAVs can gather/transmit data from sensing, monitoring, filming, etc. It is predicted that the variety of services offered by UAVs will increase exponentially in the near future [6], [7], which poses enormous challenges for future design and deployment of UAV system. Therefore, it is urgently required to develop a general traffic flow model to predict the traffic flows of the multi-services oriented UAV system for supporting extensive applications.

Only some initial works have dedicated to studying the traffic flow models. These existing traffic models can be categorized into non-self-similar models [8] and self-similar models [9], [10]. The non-self-similar traffic models focus on an ideal scenario without bursty traffic. However, the burst traffic are usually generated in various applications, such as surveillance, multimedia sensor, and habitat monitoring. In these applications, once detecting an event, a large amount of data may be generated in a short time after the event occurs, which creates a bursty traffic. On the other hand, the self-similar traffic models characterize the statistical analysis results of data collected over a long time period, and thus the network traffic exhibits the properties of self-similarity[11]. But the self-similarity traffic models cannot be employed to predict the real-time network traffic for various applications.

Manuscript received August 2, 2020; revised January 5, 2021. Corresponding author: Abderrahmane Abada, Bin Yang, and Tarik Taleb (email: \{abderrahmane.abada, bin.1.yang, tarik.taleb\}@aalto.fi).
However, the above models consider only the packet arrival rate and assume that the packets have the same structure. Thus, they cannot be applied in general network scenarios, where there exist different kinds of network services and each service has its own characteristics. For instance, the telemetry, the IoT, and the video streaming data should correspond to different network services requested by UAVs. Furthermore, the UAVs need to have different network usages in dealing with various network services with different packet arrival rate and data size. As a result, two fundamental and interrelated issues arise in UAV system. The first issue is how to jointly consider the network services and usages in designing traffic model. The second issue regards how to develop a general traffic model for multi-services oriented UAV system. Unfortunately, these two issues have not been investigated so far. In this paper ${ }^{1}$, we jointly consider these two issues in multi-services oriented UAV system. In particular, we calculate the number of packets and corresponding data size for each network service. The main contributions of this paper are summarized as follow.

- We first define the three subsets of network services requested by the UAVs. Each subset corresponds to one of the telemetry, IoT and video streaming. Under each subset, the UAVs will exhibit different usages related to packet transmission frequency and packet's size

- We use a Pareto distribution to model the non-uniformity feature of network usages. We then divide all UAVs into three subgroups depending on their network usages. By combining these three subgroups and three subsets, nine network segments can be generated, each of which consists of a subgroup and a subset.

- We further calculate the average number of packets and the total data size in each segment, the number of transmitted packets and the data size in each network

\footnotetext{
${ }^{1}$ This paper may be presented in part at the International Conference on Networking and Network Applications, Haikou, December 2020 [12].
} 
service.

- Finally, simulation results are provided to illustrate that our traffic model can well predict the number of packets and the corresponding data size for the different network services in comparison with these in real scenarios.

The rest of the paper is organized as follows. In section II, we review the related works. Section III introduce network services, subgroups for UAVs, the number of packets, and traffic data size. Case studies under these three scenarios of telemetry, IoT and streaming data are discussed in section IV. Simulation and numerical results are provided in section V. Section VI concludes this paper.

\section{RELATED WORKS}

The existing traffic models can be categorized into two categories: non-self-similar traffic models and self-similar traffic models. They use probabilistic methods to estimate the traffic flow for different traffic networks. A careful selection for the models and parameters is important for support various applications with different traffic flows.

\section{A. Non-self-similar Traffic Models}

The non-self-similar traffic models mainly include Poisson, compound Poisson and Markov-modulated Poisson models, which will be discussed below.

\section{1) Poisson traffic models}

The Poisson traffic models are studied in [13]. In the Poisson traffic models, the traffic flow is a Poisson process, which is widely used for network analysis. In this process, the interarrival times are exponentially distributed with a rate parameter $\lambda$. The Poisson distribution is appropriate if the arrivals are from a large number of independent sources, referred to as Poisson sources. The Poisson traffic models considers the following two assumptions: 1) the number of sources is infinite and 2) the traffic arrival pattern is random.

2) Compound Poisson traffic models

Based on the Poisson traffic models, the compound Poisson traffic models are proposed to deliver batches of packets at once in [14]. The compound Poisson traffic models inherit the analytical benefits of the Poisson traffic models, such as memoryless, Poisson distributed aggregation of streams, and steady-state equation. Specially, the steady-state equation is still reasonably simple to calculate, although varying batch parameters for differing flows would complicate the derivation [15]. Mathematically, this model has two parameters: the arrival rate $\lambda$ and the batch parameter $\rho \in[0,1]$.

3) Markov-modulated Poisson traffic models

Because in Point process, the arrival rates vary randomly over time arise in many applications of interest, notably in communications modeling, the Markov-modulated Poisson process (MMPP) based traffic models have been extensively used for modeling these processes [16]. It is notable that it qualitatively models the time-varying arrival rate and captures some of the important correlations between the inter-arrival times while still remaining analytically tractable. The doubly stochastic Poisson process MMPP arrival rate is an m-state irreducible Markov process.

\section{B. Self-similar Traffic Models}

The self-similar traffic models consist of Fractional Brownian Motion, Chaotic maps, and Pareto distributed traffic models.

\section{1) Fractional Brownian Motion traffic models}

The Fractional Brownian Motion is used to model the traffic models in [17]. A Fractional Brownian Motion (fBm) is a continuous-time Gaussian process defined for all positive time values, with zero mean, and auto-correlation defined on the Hurst parameter [18]. One important issue is to determine the appropriate Hurst Parameters for a self-similar flow, which proves to be fairly difficult in practice, and significantly limits the application of $\mathrm{fBm}$.

2) Chaotic maps traffic models

The Chaotic maps model [19] comes to correct the issue of TCP congestion control that seems to impact self-similar processes only in the small scale with severe impact. Chaotic maps model can be seen as continuous-state Markov chains. A chaotic system with chaotic behavior always displays the following properties: initial state sensitivity, topological transitivity, and density of periodic orbits [20]. The future behavior of a chaotic system is fully determined by its initial state. Any arbitrarily tiny change in the initial state results in a totally different orbit. With these significant properties, chaos theory has wide applications in modeling the traffic flow for various network services [21].

\section{3) Pareto distributed traffic models}

The authors in [8] investigate the Pareto distribution process based traffic models. The Pareto distribution process produces independent and identically distributed (IID) interarrival times [22]. The Pareto distributions are useful modeling and predicting tools in a wide variety of socioeconomic contexts, ans it is also well suited to distribution of income fields.

\section{TRAFFIC Flow MOdEL}

As shown in Fig. 1, we consider a general traffic flow scenario in multi-services oriented UAV system, where a swarm of UAVs can provide various network services with different traffic flows. In the scenario, UAVs equipped with diverse devices can generate telemetry data, and gather IoT data and video streaming. Here, the telemetry data is used to record the information of UAVs' status and position. The UAVs first send the data to base stations, and they then forward the data to the end users. In the following, we will introduce networks services, subgroups for UAVs, packet arrival rate, and traffic data size.

\section{A. Network Services for UAVs}

According to the three kinds of data gathered from the UAVs as shown in Fig. 1, the network services can be classified into three subsets, each of which is related to a kind of data. In these subsets, the traffic data is that (1) telemetry data representing the status information of UAVs themselves like battery level, GPS coordination, vehicle's speed, etc., (2) IoT data on-board UAVs like weather, gas, lidar sensors, etc., and 


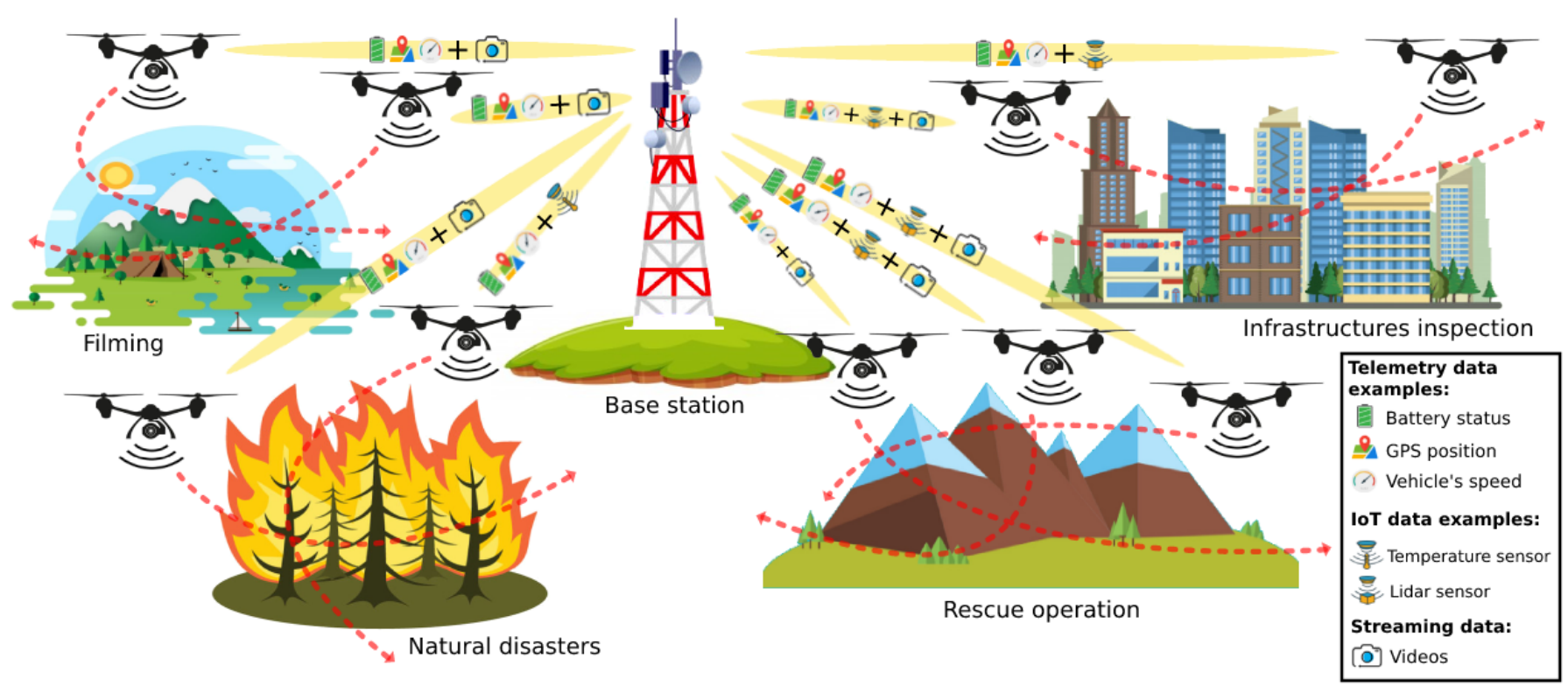

Fig. 1: A general traffic flow scenario for UAVs

(3) video streaming data captured by the cameras on-board. We use the rate of transactions denoted by $\gamma_{i}$ to represent the share of transaction for each network service subset, where $i=1,2,3$ denote the telemetry data, IoT data, and streaming data, respectively.

\section{B. Subgroups for UAVs}

According to the usages of UAVs in real world, we can classify UAVs into three subgroups: poor, middle and rich, which mean that the usage frequency of UAVs are from lowest value to highest. The poor, middle and rich are associated with the network services in terms of telemetry data, IoT data, and streaming data, respectively. We use the Gini coefficients to characterize the non-uniformity input of traffic data in these three subgroups, where the Gini coefficients are widely used to describe the non-uniformity input of population data [23]. Let $K_{G, i}$ denote the Gini coefficient, where $i=1,2,3$ and the parameter $i$ corresponds to the $i$ th subset of network services. We further consider the non-uniformity distribution as Pareto law, and then we have

$$
\alpha_{i}=\frac{0.5\left(K_{G, i}+1\right)}{K_{G, i}},
$$

where $\alpha_{i}$ denotes the Pareto parameter for the $i$ th UAV's subgroup.

We use $F_{j}$ to denote the number of UAVs in the $j$ th subgroup, where $j=1,2,3$ represent the poor, middle and rich subgroups, respectively. Lorenz curves corresponding to the Pareto distribution with parameter $\alpha$ may be written as follow [24]

$$
Q(\alpha, x)=1-(a-F(x))^{\frac{\alpha-1}{\alpha}}
$$

We use $\sigma_{i}$ to denote the percentage of network usage produced by the $i$ th subgroup for the $i$ th network service. Then, using the Lorenz curves, the subgroups can be determined as [25]

$$
F_{3}=\sigma_{3}^{\frac{\alpha_{3}}{\alpha_{3}-1}}
$$

$$
F_{2}=\sigma_{2}^{\frac{\alpha_{2}}{\alpha_{2}-1}}-F_{3}
$$

and

$$
F_{1}=1-F_{2}-F_{3} .
$$

\section{Number of Packets}

After these UAVs are divided into the three subgroups according to the network services and network usages, we obtain the following two classes of events. The first class holds the events denoted by index $i=1,2,3$ corresponding to the demands of services from the telemetry, IoT and streaming data, respectively. The events in the second class, which are denoted by index $j=1,2,3$, correspond to the demands from UAVs in the poor, middle and rich subgroups, respectively. The intersection of events from these two classes generates the segregation of nine segments. We first calculate the packet arrival rate $\lambda_{i j}$ per UAV for the all segments, and determine the number of packets generated by a swarm of UAVs.

We use $\beta_{i j}$ to denote the share of transactions related to UAVs from the $j$ th subgroup when requesting services from the $i$ th network subset. Values of $\beta_{i j}$ may be defined by two different ways that give the possibility to form the required equations bellow.

1) The definition of $\beta_{i j}$ on basis of the non-uniformity of services' requests

Values of $\beta_{i j}$ should be calculated for the nine segments. Taking into consideration the expressions $1,2,3,4,5$, and that $\sum_{k} \beta_{i j}=1$, we have

$$
\begin{aligned}
& \beta_{i 1}=1-\left(1-F_{1}\right)^{\frac{\alpha_{i}-1}{\alpha_{i}}}, \\
& \beta_{i 2}=1-\left(1-F_{1}-F_{2}\right)^{\frac{\alpha_{i}-1}{\alpha_{i}}}-\beta_{i 1}, \\
& \beta_{i 3}=1-\beta_{i 1}-\beta_{i 2},
\end{aligned}
$$


2) The definition of $\beta_{i j}$ over the rate of transaction $\lambda_{i j}$

Using the rate of transactions $\lambda_{i j}$ we can easily get the expression for the rate of transactions generated by all users of the $j$ th subgroup when requesting services from the $i$ th subset as follows $F_{j} N \lambda_{i j}$, where $N$ is the total number of UAVs. Then, $\beta_{i j}$ is determined as

$$
\beta_{i j}=\frac{F_{j} \lambda_{i j}}{\sum_{j} \lambda_{i j} F_{j}} .
$$

We now calculate the unknown parameter $\lambda_{i j}$ in (7). Given the initial input data value of $\lambda_{11}$ representing the average packet arrival rate for the telemetry data from a UAV of the poor subgroup, we have

$$
\begin{aligned}
\lambda_{12} & =\frac{\lambda_{11} \beta_{12} F_{1}}{\beta_{11} F_{2}}, \\
\lambda_{13} & =\frac{\lambda_{11} \beta_{13} F_{1}}{\beta_{11} F_{3}}, \\
\lambda_{i j} & =\frac{\gamma_{i} \beta_{i j} \sum_{j} \lambda_{1 j} F_{j}}{\gamma_{1} F_{j}}, i=2,3 ; j=1,2,3 .
\end{aligned}
$$

Let $N$ denotes the total number of UAVs in experiment area and $T$ the experiment duration in seconds. We can calculate the total number of transmitted packets $P_{i}$ in $i$ th network service subset as follows.

$$
P_{i}=N \times T \sum_{j} \lambda_{i j} F_{j}
$$

\section{Traffic Data Size}

Let $W_{i}$ denotes the average data size of a transaction from the $i$ th network service subset. We can calculate the traffic data size from the transactions rate in each network subset as follows.

$$
D_{i}=N \times T \times W_{i} \sum_{j} \lambda_{i j} F_{j} .
$$

The total traffic data size is then determined as

$$
D=N \times T \sum_{i} W_{i} \sum_{j} \lambda_{i j} F_{j}
$$

\section{CAse Study}

In this section, we present three types of cases including weather, infrastructure inspection, and research and rescue scenarios.

\section{A. Weather Scenario}

In this scenario, we aim to use UAVs to collect the temperature and humidity information in a given region. Thus, we can deploy a swarm of UAVs in the different positions of the region to get these measurements, and these UAVs are equipped with temperature and humidity sensors. As shown in Fig. 2, we provide an example of the deployment of UAVs that are divided into three subgroups. The red, green, and black UAVs represent UAVs from the first, second, and third subgroups, respectively. UAVs in the first subgroup are used to collect humidity and temperature measurement in specific places. These UAVs will be firstly sent to the position where we want to get the measurement and then send the information. UAVs in the second subgroup are average network users, they use the network services moderately to send weather measurement in different places. The UAVs in the third subgroup conduct a heavy usage of the network services, they are used to collect weather measurement in all places along their path.

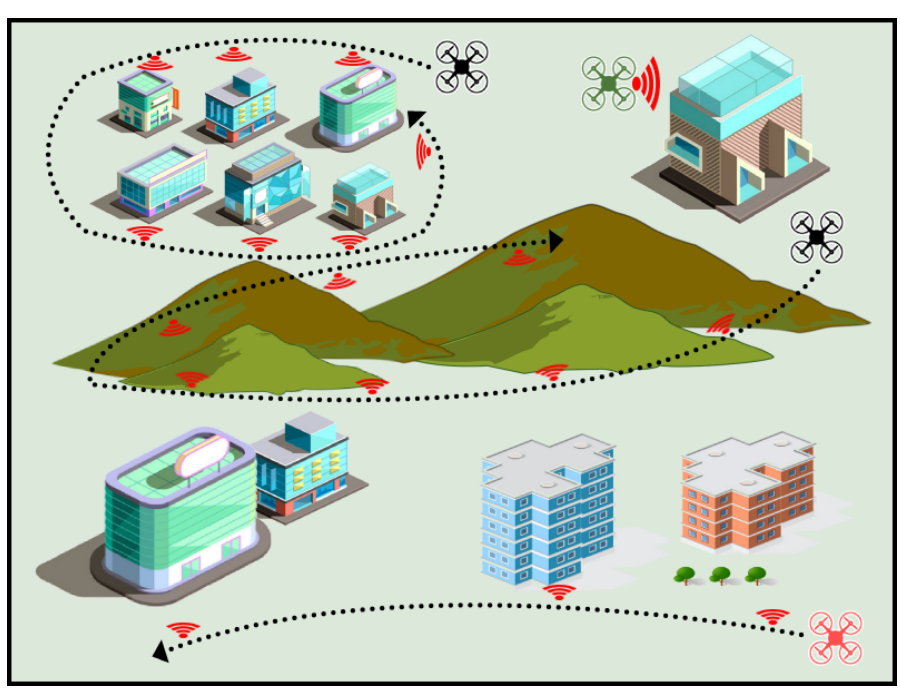

Fig. 2: Weather information's scenario

\section{B. Infrastructure Inspection Scenario}

In this scenario, we aim to inspect some infrastructures in a specific region. For this purpose, we will use a swarm of UAVs to get lidar measurements and video streaming of the premises. The UAVs will be equipped with lidar sensors and high definition cameras. The Fig. 3 shows an example of UAVs usage for this mission. UAVs in red, green, and black represent UAVs from the first, the second, and the third subgroups, respectively. UAVs from the first subgroup will be used to get lidar data and video streaming of a specific side of the premise. UAVs in the second subgroup will get measurement and video streaming for specific premises or specific sides of multiple buildings. UAVs in the third subgroup will collect lidar measurement and video streaming data for many building and from all sides.

\section{Search and Rescue Scenario}

In this scenario, multiple UAVs can be applied in search and rescue scenario. In such a scenario, UAVs equipped with thermal infrared sensors are able to locate people, and obtain their stream videos using high definition cameras. As shown in Fig. 4, we provide an example of the deployment of UAVs. UAVs in red, green, and black represent UAVs from the first, the second, and the third subgroups, respectively. The UAVs in these subgroups will perform the following operations: obtaining stream videos for the locations of people in the first subgroup, getting thermal data and stream video for the locations in the second subgroup, and getting thermal data in all region searching for people in the third subgroup. The different subgroups are performing the same scenario to ensure 


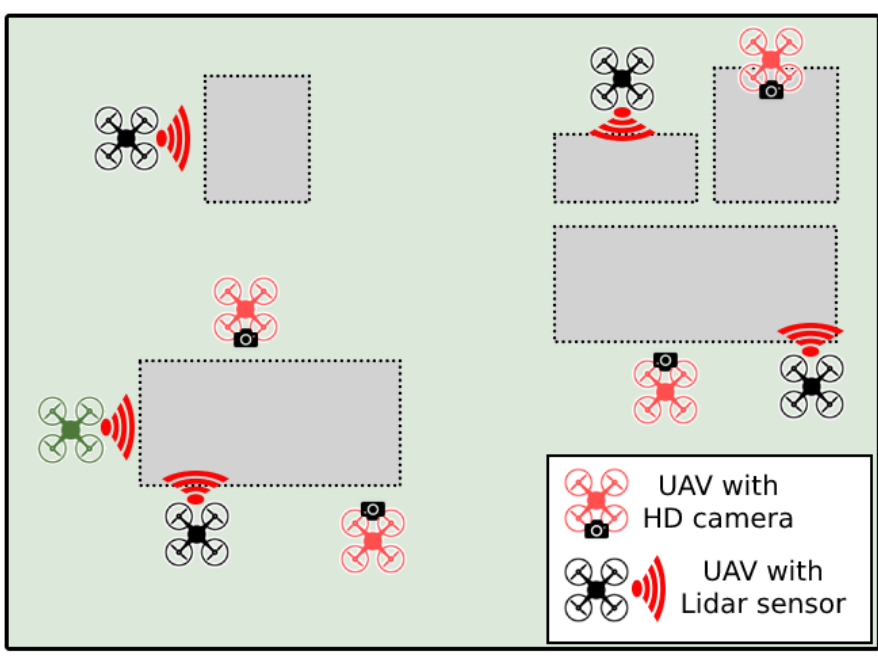

Fig. 3: Infrastructures inspection scenario

the continuity of the mission, we may want the UAVs of the third subgroup to continue their mission of searching for other people rather than stopping in the people's locations to send video streams. We use other UAVs from the first and second subgroups to perform this specific action.

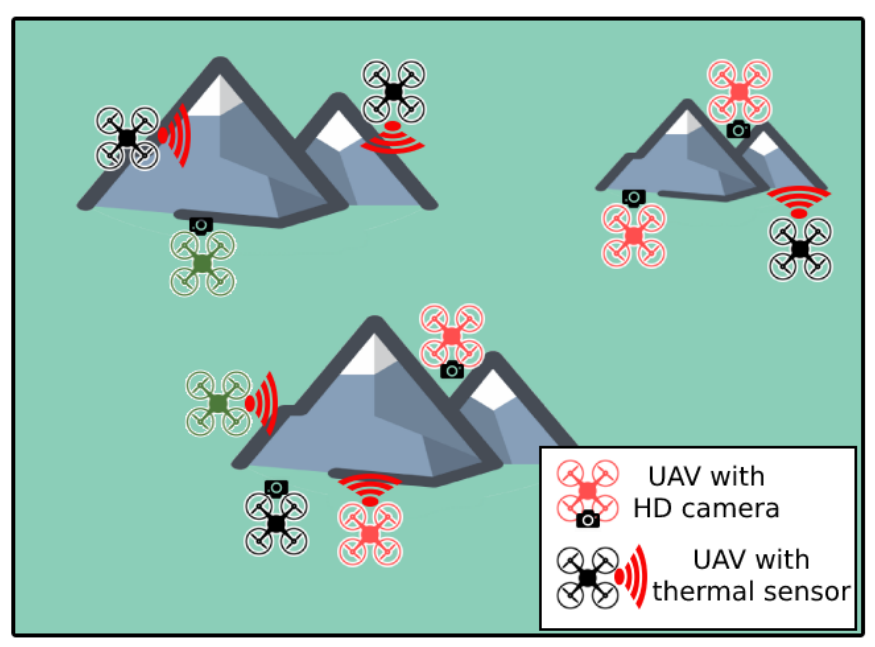

Fig. 4: Search and rescue scenario

\section{Performance Evaluation}

Based on the above traffic model and corresponding data rate formulas, this section conducts the performance evaluation study under these three scenarios illustrated in Section IV.

\section{A. Simulation Environment}

In our study, we use Software In The Loop (SITL) with Ardupilot to simulate the behaviour of the UAVs in the network. This will allow us to interact with UAVs and listen for their telemetry packets. There are $20 \mathrm{UAVs}$ distributed in the network scenarios, and their telemetry data can be sent to a server using the MAVLink protocol. On the other hand, the
IoT data like temperature, humidity and lidar are sent to the server using AMQP protocol. For the video streaming, we use a raspberry pi to stream a high definition 1080p videos. Notice that simulating multiple video streaming simultaneously on the same unit will reduce the streaming rate. To overcome this problem, we use the unit to behave as only one video streamer at a time, we run the streaming process on the unit as many time as much UAVs we simulate. In our simulation, we use 20 instances of simulated UAVs to generate telemetry data. With each UAV instance, we start a process of generating IoT data for this UAV. For the video streaming, we run 20 independent process of video streaming on the raspberry pi unit, each one corresponds to one UAV.

In the server side, we use a Python script to receive the telemetry packets, the script calculate the number of received packets and sum their lengths to calculate the data gathered from each UAV separately. The IoT data can be captured by another script, which reads all the information transmitted through the AMQP protocol and calculates the total size of packets. A script can also listen to video steams transmitted through Web sockets protocol for each UAV separately, and calculates the number of video streams and the total size of all videos sent from the unit. The parameters used in the simulation are summarized in Table I.

\section{B. Performance Analysis under Weather Scenario}

In this scenario, we analyze the number of telemetry and IoT packets and the total size of these packets predicted by our proposed theoretical traffic flow model. These packets are generated by a swarm of UAVs performing the data collection for temperature and humidity information in a specific area. We further conduct a comparison study between theoretical and simulated results. We summarize the total number of transmitted packets in Fig. 5(a) under theoretical and simulation cases. It can be observed from Fig. 5(a) that the number of telemetry packets is significantly more than that of IoT data. This is because the telemetry packets have higher transmission frequency than the IoT packets. The average telemetry packet rate is 100 packets per second per UAV, while the average IoT packet rate in the third subgroup which uses the network the most is about 11 packets per second per UAV in this scenario.

We also see from Fig. 5(b) that the total data size of telemetry packets is not significantly more than that of IoT packets. This is due to the fact that the average size of an IoT packet is much greater than the average size of a telemetry packet.

A careful observation from Figs. 5(a) and 5(b) shows that theoretical results are almost the same as the simulated ones for the number of telemetry and IoT packets and data size, respectively. This indicates that our proposed traffic flow mode for UAVs can well predict the real results.

\section{Performance Analysis under Infrastructure Inspection Scenario}

In this scenario, we investigate the number of telemetry, IoT, and streaming packets and the total size of these packets under our proposed theoretical traffic flow model and simulation one. 
TABLE I: Parameter settings

\begin{tabular}{|c|c|c|c|c|}
\hline Params. & Telem. & IoT & Stream & Description \\
\hline$\alpha_{i}$ & 10 & 1.125 & 1.06 & Pareto coefficient \\
\hline \multirow{3}{*}{$\gamma_{i}$} & $98 \%$ & $2 \%$ & & $\begin{array}{l}\text { Share of } \\
\text { transactions for } \\
\text { weather scenario }\end{array}$ \\
\hline & $98 \%$ & $1.9 \%$ & $0.1 \%$ & $\begin{array}{l}\text { Share of } \\
\text { transactions for } \\
\text { infrastructure } \\
\text { inspection } \\
\text { scenario }\end{array}$ \\
\hline & $96 \%$ & $3.9 \%$ & $0.1 \%$ & $\begin{array}{l}\text { Share of } \\
\text { transactions for } \\
\text { search and } \\
\text { rescue scenario }\end{array}$ \\
\hline$W_{i}$ & $34 \mathrm{~B}$ & 1240B & $1 \mathrm{MB}$ & $\begin{array}{l}\text { Average size of } \\
\text { a transaction }\end{array}$ \\
\hline $\mathrm{N}$ & \multicolumn{3}{|l|}{20} & $\begin{array}{l}\text { Total number of } \\
\text { used UAVs }\end{array}$ \\
\hline $\mathrm{T}$ & \multicolumn{3}{|l|}{$60 \mathrm{~s}$} & $\begin{array}{l}\text { Observation } \\
\text { duration in } \\
\text { seconds }\end{array}$ \\
\hline$\sigma_{3}$ & \multicolumn{3}{|l|}{0.9} & $\begin{array}{l}\text { The percentage } \\
\text { of network usage } \\
\text { of streaming data } \\
\text { from the } 3^{\text {rd }} \\
\text { subgroup }\end{array}$ \\
\hline$\sigma_{2}$ & \multicolumn{3}{|l|}{0.9} & $\begin{array}{l}\text { The percentage } \\
\text { of network usage } \\
\text { of IoT data from } \\
\text { the } 2^{\text {nd }} \text { and the } \\
3^{r d} \text { subgroups }\end{array}$ \\
\hline$\lambda_{11}$ & \multicolumn{3}{|c|}{$\begin{array}{l}100 \\
\text { trans/UAV }\end{array}$} & $\begin{array}{l}\text { Rate of } \\
\text { transactions of } \\
\text { the first } \\
\text { subgroup for the } \\
\text { first telemetry } \\
\text { data }\end{array}$ \\
\hline
\end{tabular}

These packets consist of lidar information and video streams of the premises from an inspection of some infrastructures in a given area conducted by a swarm of UAVs. We summarize the total number of transmitted packets in Fig. 6(a) under theoretical and simulation cases. We can see from Fig. 6(a) that the number of generated streaming packets is extremely few compared with IoT and telemetry packets. This is because the average streaming packet rate in the third subgroup is very low approximating 0.63 packet per second per UAV in this scenario.

It can be seen from from Fig. 6(b) that the total data size of generated streaming packets represent approximately $95 \%$ of the total transmitted data. This is because the average size of one video is much greater than the average size of a telemetry and IoT packets.

We further observe from Fig. 6 that theoretical results are

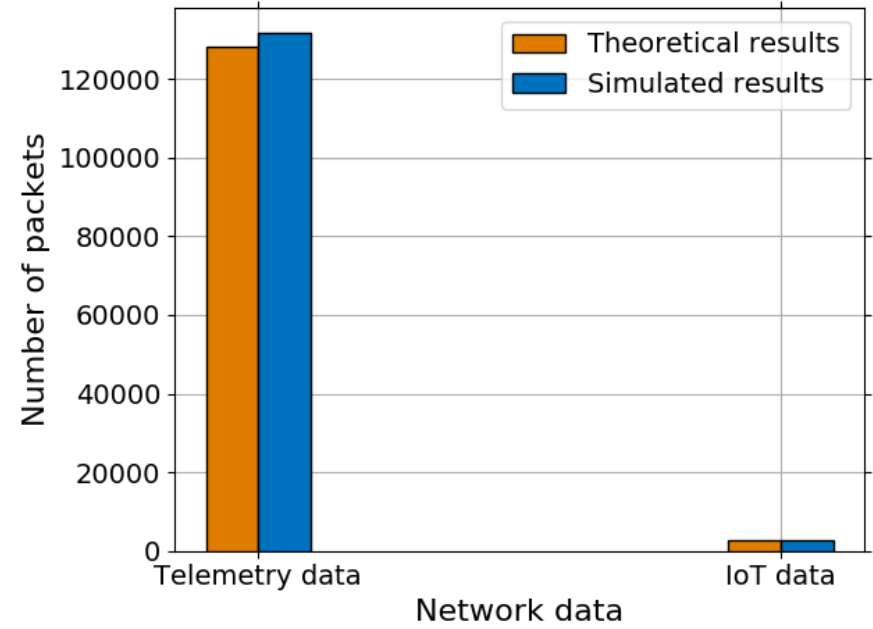

(a) Number of transmitted packets

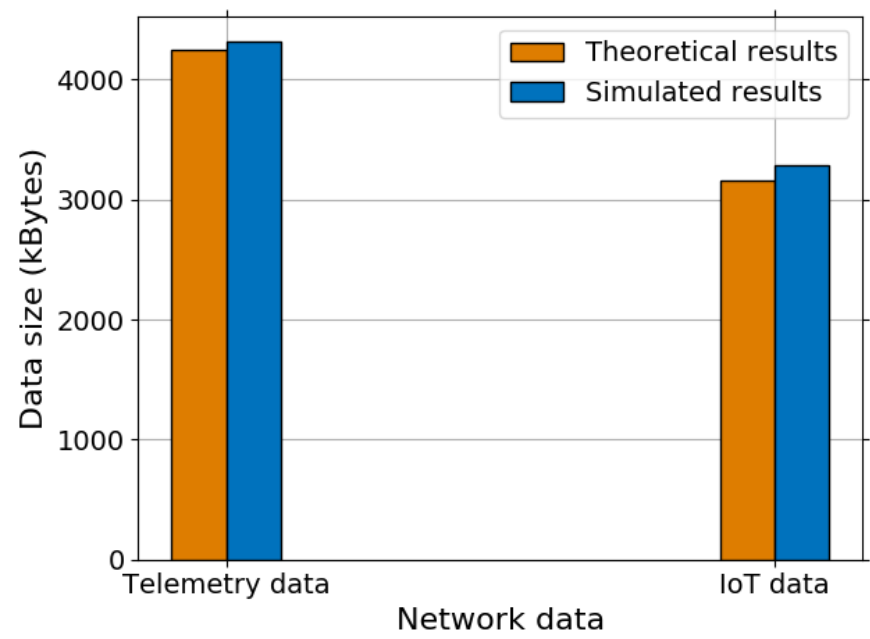

(b) Network data usage

Fig. 5: Number of transmitted packets and network data usage under the weather scenario

almost the same as the simulated ones for the number of telemetry, IoT, and streaming packets and for the data size, respectively. This also indicates that our proposed traffic flow model for UAVs can also predict the real streaming video data.

\section{Performance Analysis under Search and Rescue Scenario}

In this scenario, we investigate how the extensive use of the on-board IoT affect the number of telemetry, IoT, and streaming packets, and the total size of these packets under search and rescue scenario in a given area. The search operation is to unitize the on-board thermal sensor to search for people in remote places. Once the video streams are received by the rescue team, they can obtain the people's states and locations so that further performing rescue operation.

We summarize the total number of transmitted packets in Fig. 7(a) under theoretical and simulation cases. An observation from Fig. 7(a) shows that the total number of generated telemetry packets is always more than the number of generated IoT packets. That is because even in this extensive IoT usage in this scenario, the average arrival rate for telemetry packets 


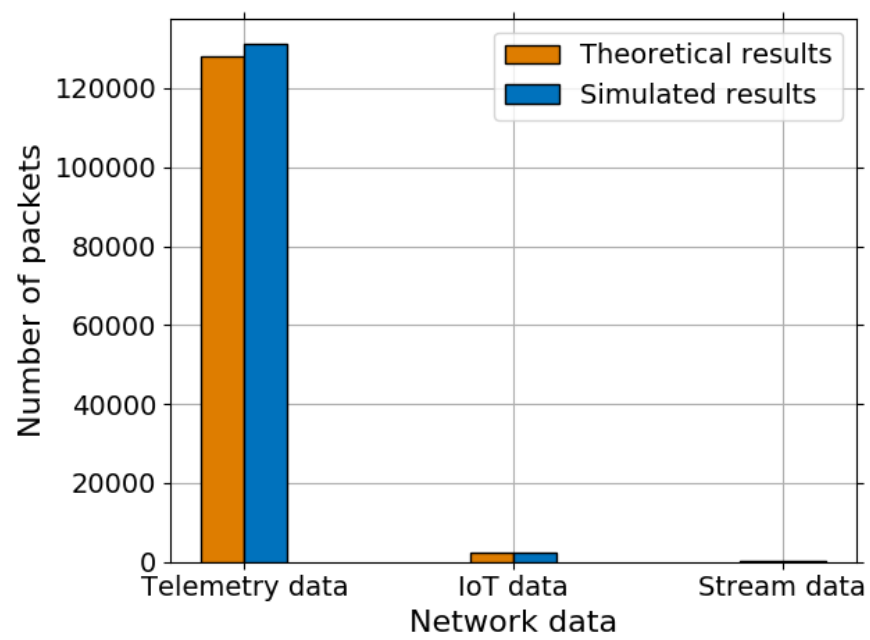

(a) Number of transmitted packets

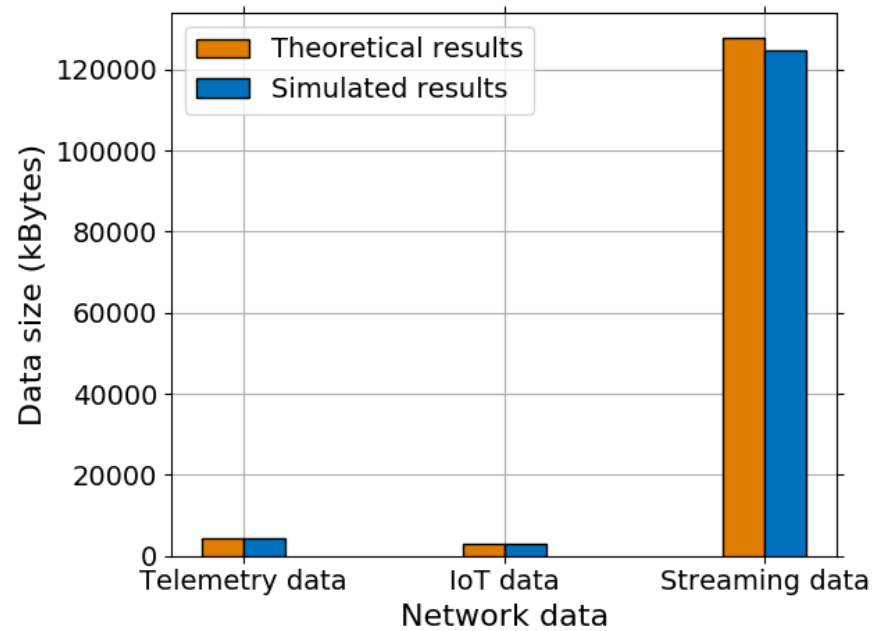

(b) Network data usage

Fig. 6: Number of transmitted packets and network data usage under the infrastructure inspection scenario

is higher than that for IoT packets. The former rate is 100 packets/s while the latter one is about 22.68 packets per second per UAV in the third subgroup.

We can see from Fig. 7(b) that the size of generated IoT packets exceeds the size of telemetry packets. This is because in this scenario, UAVs extensively use the IoT sensors onboard for search operation which leads to an increase in the network data usage for the IoT service.

\section{CONCLUSION}

In this paper, we proposed a general traffic flow model for multi-services oriented UAV system. Under such a model, the network services are first categorized into three subsets, and then the UAVs are divided into three subgroups, which forms nine mapping relationships between the subsets and subgroups. We further determined the number of packets for each service and total data size. The simulation results indicate that our proposed traffic flow model is general that it can predict the traffic flows under different scenarios like weather, infrastructure inspection, and search and rescue scenarios. These

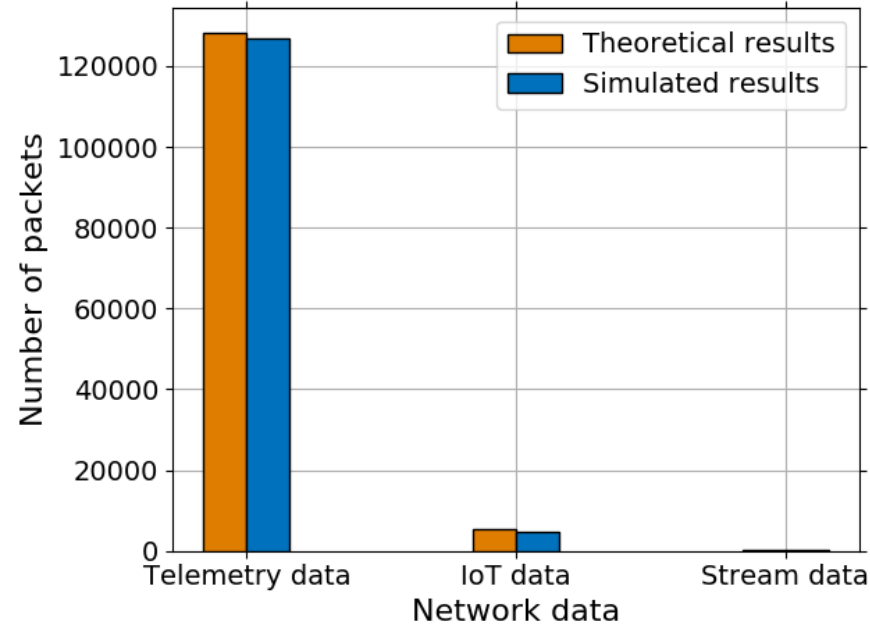

(a) Number of transmitted packets

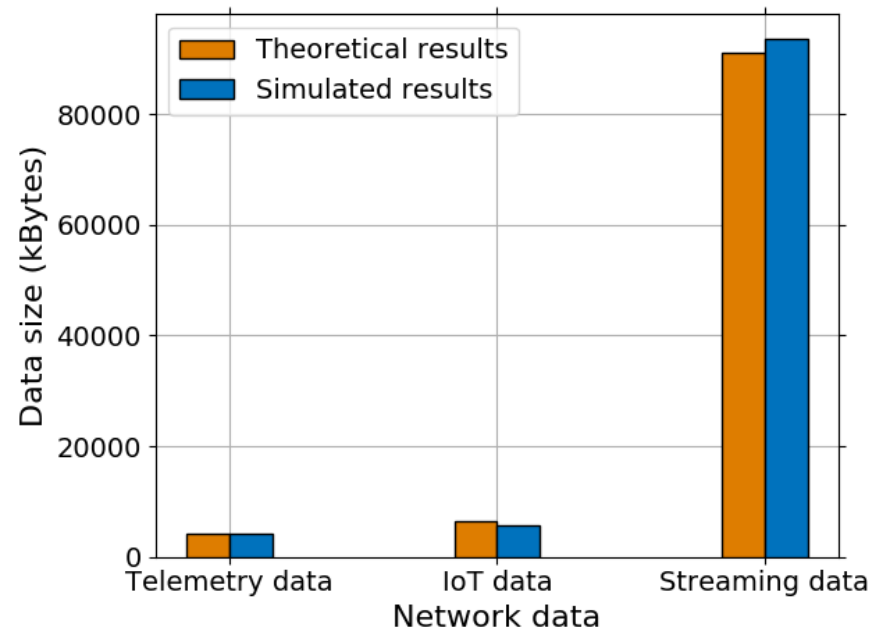

(b) Network data usage

Fig. 7: Number of transmitted packets and network data usage under the search and rescue scenario

results also reveal that most of traffic packets are generated by telemetry service while data size of streaming packets is much larger than these of telemetry and IoT packets in muti-services oriented UAV system. Our research is expected to facilitate the design and deployment of the system with various service requirements.

\section{ACKNOWLEDGEMENT}

This work was supported by the European Union's Horizon 2020 Research and Innovation Program through the 5G!Drones Project under Grant No. 857031, the Academy of Finland 6Genesis project under Grant No.318927, the Academy of Finland CSN project under Grant No. 311654, the NSF of China under Grant No. 61962033, the Anhui Province project under Grant No. 1808085MF165, gxgwfx2019060 and KJ2019A0643, the Yunnan Province project under Grant No. 2018FH001-010, and the CHZU project under Grant No. 2020qd16 and zrjz2019011. 


\section{REFERENCES}

[1] S. K. Khan, "Performance evaluation of next generation wireless uav relay with millimeter-wave in access and backhaul," 2019.

[2] N. H. Motlagh, M. Bagaa, and T. Taleb, "Energy and delay aware task assignment mechanism for UAV-based IoT platform," IEEE Internet Things J., vol. 6, no. 4, p. 6523-6536, Aug. 2019.

[3] B. Yang, T. Taleb, Z. Wu, and L. Ma, "Spectrum sharing for secrecy performance enhancement in D2D-enabled UAV networks," IEEE Network, vol. 34, no. 6, pp. 156-163, Nov./Dec. 2020.

[4] N. H. Motlagh, T. Taleb, and O. Arouk, "Low-altitude unmanned aerial vehicles-based internet of things services: Comprehensive survey and future perspectives," IEEE Internet of Things J., vol. 3, no. 6, pp. 899922, Dec. 2016.

[5] C. Benzaid and T. Taleb, "ZSM security: Threat surface and best practices," IEEE Network, vol. 34, no. 3, pp. 124-133, 2020.

[6] B. de Miguel Molina and M. S. Oña, "The drone sector in europe," in Ethics and civil drones. Springer, Cham, 2018, pp. 7-33.

[7] B. Yang, T. Taleb, Y. Shen, X. Jiang, and W. Yang, "Performance, fairness and tradeoff in uav swarm underlaid mmwave cellular networks with directional antennas," IEEE Trans. Wireless Commun., 2020. [Online]. Available: https://doi.org/10.1109/TWC.2020.3041800

[8] B. Chandrasekaran, "Survey of network traffic models," Waschington University in St. Louis CSE, vol. 567, 2009.

[9] O. Boxma and J. Cohen, "The single server queue: Heavy tails and heavy traffic," Self-Similar Network Traffic and Performance Evaluation, pp. $143-169,2000$

[10] R. R. Marie, J. M. Blackledge, and H. E. Bez, "Characterization of internet traffic using a fractal model," in Proceedings of the Fourth conference on IASTED International Conference: Signal Processing, Pattern Recognition, and Applications. ACTA Press, 2007, pp. 253258.

[11] M. Grossglauser and J.-C. Bolot, "On the relevance of long-range dependence in network traffic," IEEE/ACM transactions on networking, vol. 7, no. 5, pp. 629-640, 1999.

[12] A. Abada, B. Yang, and T. Taleb, "Traffic flow modeling for UAVenabled wireless networks," Submitted to the 2020 International Conference on Networking and Network Applications.

[13] M. Becchi, "From poisson processes to self-similarity: a survey of network traffic models," Washington University in St. Louis, Tech. Rep, 2008.

[14] R. Jain and S. Routhier, "Packet trains-measurements and a new model for computer network traffic," IEEE journal on selected areas in Communications, vol. 4, no. 6, pp. 986-995, 1986.

[15] M. Wilson, "A historical view of network traffic models," Unpublished survey paper. See http://www. arl. wustl. edu/mlw2/classpubs/traffic models, 2006.

[16] W. Fischer and K. Meier-Hellstern, "The markov-modulated poisson process (mmpp) cookbook," Performance evaluation, vol. 18, no. 2, pp. 149-171, 1993.

[17] J. L. Véhel and R. Riedi, "Fractional brownian motion and data traffic modeling: The other end of the spectrum," in Fractals in engineering. Springer, 1997, pp. 185-202.

[18] P. Flandrin, "Wavelet analysis and synthesis of fractional brownian motion," IEEE Transactions on information theory, vol. 38, no. 2, pp. 910-917, 1992.

[19] P. Pruthi and A. Erramilli, "Heavy-tailed on/off source behavior and self-similar traffic," in Proceedings IEEE International Conference on Communications ICC'95, vol. 1. IEEE, 1995, pp. 445-450.

[20] B. Hasselblatt and A. Katok, A first course in dynamics: with a panorama of recent developments. Cambridge University Press, 2003.

[21] Z. Hua and Y. Zhou, "Nonlinear chaotic processing model," arXiv preprint arXiv:1612.05154, 2016.

[22] J. Gordon, "Pareto process as a model of self-similar packet traffic," in Proceedings of GLOBECOM'95, vol. 3. IEEE, 1995, pp. 2232-2236.

[23] P. M. Dixon, J. Weiner, T. Mitchell-Olds, and R. Woodley, "Bootstrapping the gini coefficient of inequality," Ecology, vol. 68, no. 5, pp. 1548$1551,1987$.

[24] L. Varakin, "The pareto law and the rule 20/80: the distribution of incomes and telecommunication services," MAC proceedings, vol. 1, pp. $3-10,1997$.

[25] A. Krendzel, Y. Koucheryavy, J. Harju, and S. Lopatin, "Method for estimating parameters of $3 \mathrm{~g}$ data traffic," in 2004 IEEE International Conference on Communications (IEEE Cat. No. 04CH37577), vol. 7. IEEE, 2004, pp. 4312-4316.

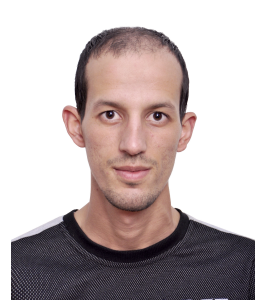

Abedrrahmane Abada received his engineer's degree in IT systems and software from the Higher National School of Computer Science, Algiers, Algeria in 2018. He is currently pursuing his Ph.D degree in the School of Electrical Engineering, Aalto University, Espoo, Finland. His field of research focuses on UAV communication and control using $5 \mathrm{G}$ network.

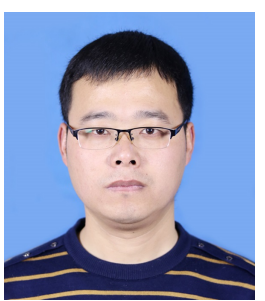

Bin Yang received the Ph.D. degree in systems information science from Future University Hakodate, Japan in 2015. He is a professor with the School of Computer and Information Engineering, Chuzhou University, China, and is also a research fellow with the School of Electrical Engineering, Aalto University, Finland. His research interests include unmanned aerial vehicle networks, cyber security and Internet of Things.

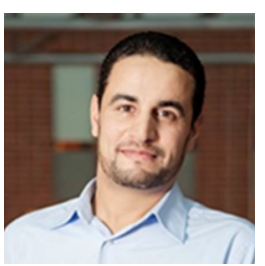

Tarik Taleb received the B.E. degree (with distinction) in information engineering in 2001, and the M.Sc. and Ph.D. degrees in information sciences from Tohoku University, Sendai, Japan, in 2003, and 2005, respectively. He is currently a Professor with the School of Electrical Engineering, Aalto University, Espoo, Finland. He is the founder and the Director of the MOSA!C Lab. He is the Guest Editor-in-Chief for the IEEE JSAC series on network softwarization and enablers. 